\title{
ACTIVE SOI-BASED NEURAL PROBES
}

\section{Matthew D. Hills ${ }^{1}$, David T. Kewley ${ }^{2}$, James M. Bower ${ }^{2,3}$, Gregory T. A. Kovacs ${ }^{1}$}

\author{
(1) Department of Electrical Engineering \\ Stanford University \\ Stanford, CA 94305-4075, USA \\ (2) Computation and Neural Systems \\ California Institute of Technology \\ Pasadena, CA 91125, USA
}

\author{
(3) currently with the Research Imaging Center \\ University of Texas Health Science Center \\ and the Cajal Neuroscience Center \\ University of Texas at San Antonio \\ San Antonio, TX 78249, USA
}

\begin{abstract}
This paper reports in vivo recordings from a new integrated, micromachined neural probe. The probes were fabricated by combining a standard CMOS process with a decoupled probeformation process on silicon-on-insulator wafers. The fabricated probes include thirty-two electrode recording sites with low noise buffering to yield a high signal-to-noise ratio. A subthreshold-biased filtering system to cancel electrode-related offsets is also presented.
\end{abstract}

\section{INTRODUCTION}

Silicon-based probes are a unique tool for neurophysiologists studying the interactions of multiple neurons, where precise electrode placement is critical. Researchers have begun to realize these benefits [1] as passive silicon-based, multi-electrode arrays have started to become available to the neurophysiology research community.

Neural probes must be designed to minimize perturbations to the biological system, which requires biocompatible materials and reducing tissue trauma through smooth, low-profile shafts [2]. Electrically, the neural probe system must be capable of recording low-level extracellular action potentials, with frequencies of interest ranging from $100 \mathrm{~Hz}-10 \mathrm{kHz}$ and amplitudes of $50-500 \mu \mathrm{V}$, and field potentials, with frequencies of interest from $1 \mathrm{~Hz}-100 \mathrm{~Hz}$ and amplitudes up to a few $\mathrm{mV}$.

By integrating such probes with circuitry, signal quality can be improved by buffering the signals on-chip, which minimizes parasitic losses due to wiring and packaging and reduces susceptibility to crosstalk and noise pick-up. Early active probe work using PMOS or NMOS processes $[\mathbf{3 , 4}]$ demonstrated the potential for integrating circuits on the probes. More recent active probe work has shifted towards integrating with a custom CMOS process and has achieved higher yields [5]. This custom CMOS process is based upon epitaxial wafers, requires preliminary deep Boron implants to establish the etch stop that defines the probe shape, and requires special care to avoid undercutting the circuitry.

Electrodes exhibit a time-varying drift in the electrode potential [6] that can saturate high-gain amplifiers and degrade the performance of differential amplifiers. Conventional recording systems use discrete high-pass filters at the front end to block these offsets. Large capacitors have been integrated on-chip for this purpose [3], but this procedure is not area efficient for multiple channels. Other approaches include reset switches, which suffer from feedthrough, and diodes, which are not reliably able to prevent amplifier saturation [5].

The probe design presented here addresses these issues through a fabrication procedure that decouples the probe-formation process from the standard CMOS process, and the implementation of electrode offset-cancellation circuitry with low-noise buffering.

\section{SYSTEM DESIGN}

The current probes are fork-shaped structures with a full wafer thickness base area and multiple, thin shafts that penetrate the cortex (Figure 1). In designing the active probes, the primary goal was to develop a reliable CMOS-compatible process to facilitate simultaneous recording of a large number of high-quality signals. In order to achieve this, it was necessary to use area-efficient, lownoise buffering circuitry and to develop a probe shaping process that would not require modifications to the underlying circuit process.

Probe Design. The probe formation includes the overall definition of the probe shaping, the electrodes, and the traces. Probe shaping is accomplished through plasma etches stopping on the oxide of a silicon-on-insulator (SOI) wafer. Plasma etching offers flexible control over the probe geometries [7], while sidewall smoothness and shape can be controlled by the etch recipe [8]. Overall shaft thickness is set by the $25 \mu \mathrm{m}$ device layer, allowing for good rigidity and strength for the 3-5mm shaft lengths. "Bond and etchback" SOI wafers are used to provide the relatively thick device layer and a smooth oxide interface, which translates into a smooth underside on the shafts. Similar probe-shaping methods have recently been reported by several investigators developing passive probes $[\mathbf{9 , 1 0}]$.

The recording electrodes were $100 \mu \mathrm{m}^{2}$ in area and metallized with evaporated gold. The geometry balances the selectivity of small recording sites [11] against the lower source impedances of larger sites, while the gold electrode is resistant to corrosion [12] and is readily available for evaporation.

The second metal (aluminum) layer from the CMOS process was used to form the traces along the shafts. By including a dielectric between the trace and electrode, the overall shaft width could be minimized and tetrode geometries could be readily designed. A thick barrier layer of titanium was used between the aluminum traces and gold electrodes to prevent the intermetallic formations of "purple plague."

Preliminary experiments demonstrated that the silicon substrate can also act as a parasitic electrode. In order to avoid driving this electrode, active probe designs typically tie this substrate potential to the biological reference [5]. To minimize possible loading on the system, the substrate was also encapsulated in a silicon nitride dielectric. Due to the difficulty of selectively encapsulating a three-dimensional structure, this was done by relying upon the relative anisotropies of the deposition and etch processes. Such an approach is not well-controlled and would likely have passivation failures, particularly close to the probe tips, but

Travel support has been generously provided by the Transducers Research

Foundation and by the DARPA MEMS and DARPA BioFlips programs. 


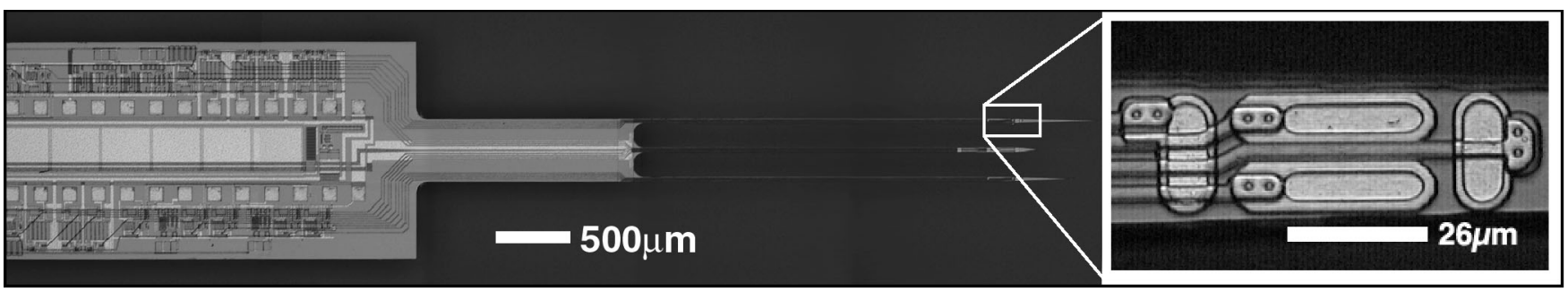

Figure 1. Micrograph of an active probe with an enlargement of a tetrode recording structure. The large capacitor structure in the center of the circuit area filters the on-chip bias. In the inset, note the narrow shaft width achieved by the multiple metal levels and anisotropic silicon etch.
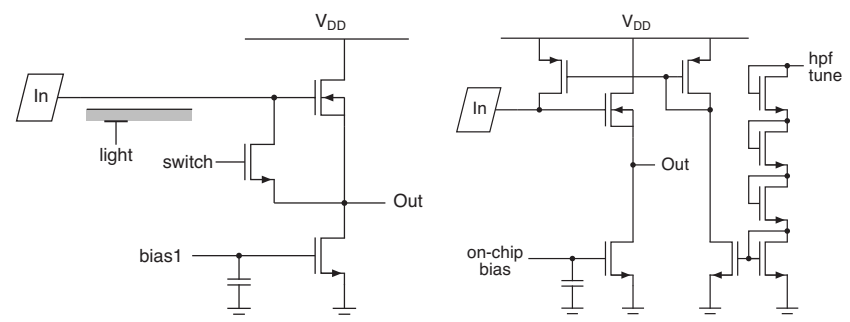

Figure 2. Schematics of the source-follower circuit (left), and the electrode offset cancellation circuit (right).

preliminary cross-sections of shafts showed good coverage that would minimize the substrate electrode area.

Light-induced noise was observed in previous work with passive probes and was determined to arise from carrier generation in depletion regions underneath the traces and electrodes [7]. This was avoided by utilizing the implants of the CMOS process to dope the shafts and then keep the traces biased so that the shafts remain in accumulation. The coupling could also have been reduced by increasing the dielectric thickness, but with tradeoffs against crosscoupling, shaft cross-sectional area, stress-induced curvature in the shafts, and constraints upon the underlying CMOS process.

To improve upon the handling and packaging issues encountered with earlier probe work [7], it was considered important to work with full-wafers throughout the process and to increase the thickness of the base underneath the circuits. The SOI substrate provides both of these features. The oxide layer provides a reliable etch stop for both front-side probe shaping, as well as the back-side release etch. Furthermore, patterning the back side prior to the release etch allows for a thick substrate by which to handle the individual probes and also improves mounting alignment by butting against an edge on the probe mount. After the release etch, the probe bases are still attached to the wafer by tabs that can later be mechanically severed when the probes are ready for packaging. After mounting and wirebonding, the probe bases are encapsulated in an opaque epoxy to protect the bondwires and circuits from the electrolyte and shield the circuitry from light effects.

Circuit Design. For small signals over the bandwidth of interest for neural recording, recording electrodes are typically modeled as high-impedance capacitive sources [13]. In order to buffer the signals most efficiently, source followers were used (Figure 2). NFET devices were selected to allow inputs at the positive rail, which was necessary for biasing the biological reference at the substrate potential in the $\mathrm{p}$-well process. With these devices, $1 / \mathrm{f}$ noise dominates the circuit noise performance and can be reduced as a function of device area. Techniques for reducing 1/f noise, such as chopper stabilization, introduce problems from clock feedthrough at

the high-impedance node. As single-ended designs are sensitive to power-supply noise, the system uses a regulated and filtered battery power supply and the on-chip bias circuit includes a large MOS capacitor to minimize noise on the shared bias line. Other buffer topologies have been demonstrated [5] that can improve the PSRR and output resistance, but with typical trade-offs against die area, noise performance, and power.

The electrode offset cancellation is accomplished through a tunable high pass filter formed by the electrode and a variablybiased sub-threshold device (Figure 2). Earlier work [7] demonstrated the utility of sub-threshold devices for implementing tunable, low-frequency, high-pass filters between stages to prevent amplifier saturation. The current design moves this to the front of the first stage in order to prevent noise degradation resulting from the dc offsets unbalancing the amplifier input. A similar technique was prototyped [14], although implementation required trimming a resistor to compensate for process variations and was not integrated with a probe.

\section{PROBE FABRICATION}

The integrated probes were fabricated on 4-inch BESOI wafers with a $25 \mu \mathrm{m}$ thick, $\langle 100\rangle$ n-type device layer (SiBond L.L.C., Hopewell Junction, NY). Processing proceeded in three phases (Figure 3), and then the probes were mounted on pc boards for use.

Circuit Fabrication. The circuit fabrication followed a standard p-well, poly-gate CMOS sequence [15]. The $n+$ substrate contact was implanted over the shafts as the light shield. The field oxide and first layer of low temperature oxide (LTO) were removed from non-circuit areas during processing by the active and contact etches, respectively. The second $600 \mathrm{~nm}$ LTO layer was deposited and patterned for vias and with the outline of the probe, so that it would form the dielectric underneath the probe traces. Metal two was sputter-deposited Aluminum 700nm followed by $50 \mathrm{~nm}$ of titanium, and was patterned with a $\mathrm{Cl}_{2} / \mathrm{BCl}_{3}$ plasma etch.

Electrode Formation. Metal two was encapsulated by $600 \mathrm{~nm}$ of PECVD silicon nitride deposited at $350^{\circ} \mathrm{C}$. This was patterned using a plasma etch with $\mathrm{SF}_{6}$ and $\mathrm{CF}_{3} \mathrm{Br}$ to clear the contacts for the electrodes and also clear the outline of the probe. To define the electrode areas, a liftoff structure was created using NR8-3000 (Futurrex, Inc., Franklin, NJ), where the undercut necessary for the liftoff was adjusted via the lithography exposure energy. For the electrode, $100 \mathrm{~nm}$ of titanium, followed by $650 \mathrm{~nm}$ of gold were deposited by ebeam evaporation. The liftoff was conducted in EMT$130 \mathrm{~T}$ resist stripper heated to $80^{\circ} \mathrm{C}$, and the wafers were then rinsed in a mask cleaner to fully remove residual electrode material from the wafer edges. The $1 \mu \mathrm{m}$ silicon nitride upper dielectric was deposited 


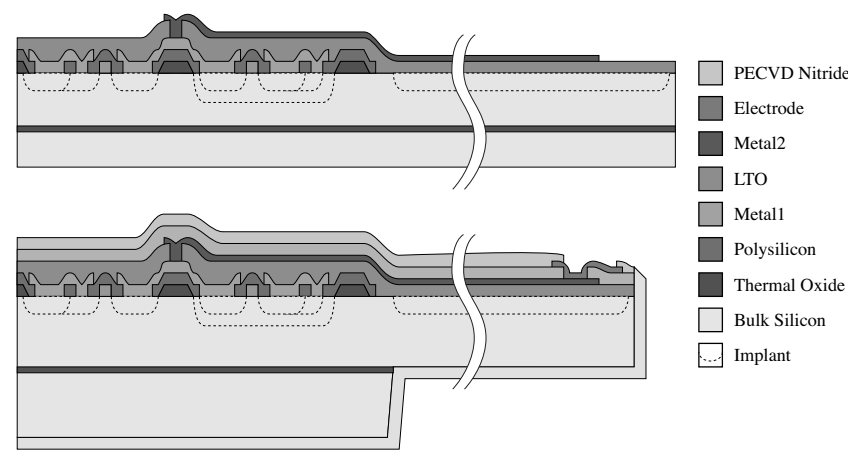

Figure 3. Cross-sections of probe during processing. The top diagram shows the probe after completion of CMOS through top metal. The bottom diagram shows a cross-section after electrode metallization, passivation, and probe shaping.

by $\mathrm{PECVD}$ at $350^{\circ} \mathrm{C}$ and then patterned using an $\mathrm{SF}_{6} / \mathrm{CF}_{3} \mathrm{Br}$ plasma etch to define the electrode openings, bondpads, and the overall outline of the probe. In order to help protect the dielectrics, a $30 \mathrm{~nm}$ layer of chromium was sputter deposited and patterned using CR-14 Chromium Etch (Cyantek Corp., Fremont, CA).

Probe Formation. The probe shape was defined using a deep reactive ion etch (DRIE etch) [8] that utilized switched $\mathrm{SF}_{6}$ and $\mathrm{C}_{4} \mathrm{~F}_{8}$ gas flows. As the front-side etch neared completion, the switching ratio was biased to provide more passivation, in order to minimize the lateral etching at the buried oxide layer characteristic of the Bosch etch process [16]. The front side of the wafer was then coated with a thick layer of AZP4620 photoresist (Clariant Corp., Somerville, NJ) as protection during the back-side patterning. The back side was patterned with a back-side mask aligner aligning against the front-side stepper-generated fields. This registration was within the $\pm 10 \mu \mathrm{m}$ tolerances in the design layout. The residual oxide and nitride on the back of the wafer were removed using a $\mathrm{CHF}_{3} / \mathrm{O}_{2}$ plasma etch, and then the back-side silicon was etched using a DRIE etch. The buried oxide was removed with a $\mathrm{CHF}_{3} / \mathrm{O}_{2}$ plasma etch. After stripping resist and cleaning the wafers, $500 \mathrm{~nm}$ of silicon nitride was deposited on the back side of the probes. The front side of the wafers was then etched in an $\mathrm{SF}_{6} / \mathrm{CF}_{3} \mathrm{Br}$ plasma to remove the substrate-protection nitride that deposited on the probe fronts. The chromium was removed with a quick dip in 50:1 HF to remove chromium oxide, followed by an etch in a 1:1 mixture of acetic acid and CR-14 Chromium Etch, then the probes were rinsed and dried at $150^{\circ} \mathrm{C}$.

Mounting. The probes were separated from the wafer by cleaving the tabs to the bulk wafer. A low-stick tape was used underneath the bases to hold them in place. The probes were positioned on the carrier pc boards by butting them against the edge of the alignment groove and bonding with epoxy. Electrical connections were made by wirebonding to the pc board, and the base and wirebond area was encapsulated in Epo-Tek 87GT epoxy (Epoxy Technology, Inc., Billerica, MA).

\section{EXPERIMENTAL RESULTS}

After the initial CMOS device verification, the probes were first tested in vitro to quantitatively measure system performance and then tested in vivo to verify the performance for the intended application. The passive probes created in the same process run

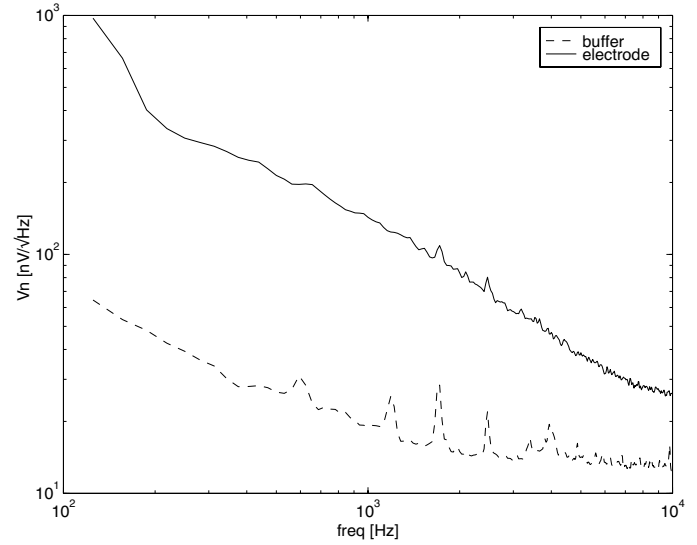

Figure 4. Spectral plots showing the noise from a buffer with grounded inputs and from a buffer with a 100 $\mathrm{mm}^{2}$ electrode in grounded saline. Note that the electrode noise dominates.

have been employed for other physiology experiments that have been presented separately [17].

Benchtop Testing. The source followers occupy $0.05 \mathrm{~mm}^{2}$ and dissipate $50 \mu \mathrm{W}$ per channel. Their output impedance is $7 \mathrm{k} \Omega$ with a grounded input, and $13.4 \mathrm{k} \Omega$ with a $100 \mu \mathrm{m}^{2}$ electrode at the input. $1 /$ f noise is dominant over the $100 \mathrm{~Hz}-10 \mathrm{kHz}$ frequency range, with a process $K f_{\text {NMOS }}=2.010^{-24} \mathrm{~V}^{2} \mathrm{~F}$. As shown in Figure 4, the followers alone exhibit a total noise of $1.7 \mu \mathrm{Vrms}$, while driving the followers through $100 \mu \mathrm{m}^{2}$ electrodes in grounded saline gives a total system noise of $11 \mu \mathrm{Vrms}$.

The electrode offset cancellation circuit performance is demonstrated in Figure 5, and its tuning behavior is shown in Figure 6. The effectiveness of the substrate light protection was assessed by testing sensitivity to laboratory ambient lighting in saline, as shown in Figure 7.
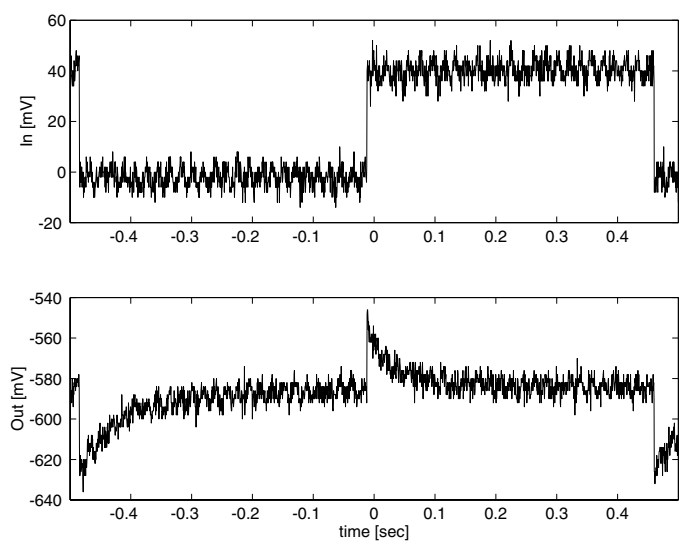

Figure 5. Response (bottom trace) of the electrode offset compensation circuit to a $50 \mathrm{mV}$ step (top trace) applied to a $\mathrm{Ag} / \mathrm{AgCl}$ electrode in saline. A $10 \mathrm{mV}$ high-frequency signal is superimposed on the input for comparison.

Neural Recordings. The subjects for the physiological tests of the probes were 40-60 day old female albino Sprague-Dawley rats. During each experiment, the animal was anesthetized with ketamine and placed in a stereotaxic rig. Surgery was performed to expose a small area over the parietal cortex, where the probe insertions were 


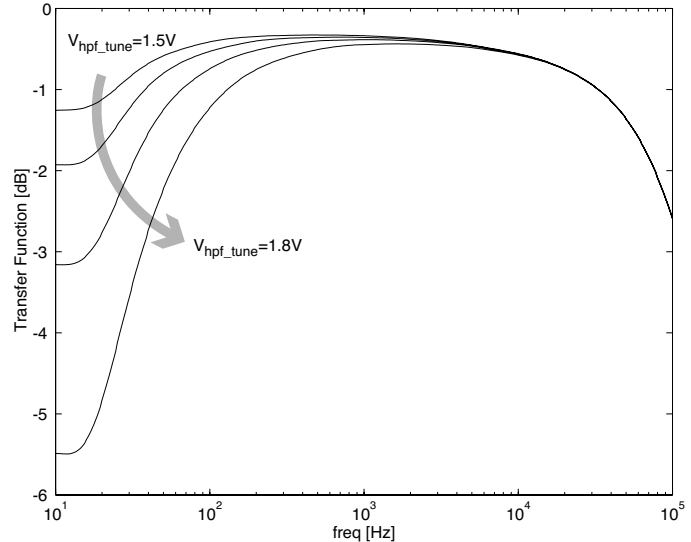

Figure 6. Transfer function of the electrode offset cancellation circuit showing the tunable high-pass characteristic.

to be performed. Figure 8 shows several channels of spontaneous neural activity recorded from parietal cortex using the active probes. Data was collected using a multi-channel amplifier and data acquisition system [18].

The signal quality is comparable to that reported from a custom CMOS process [5]. The light protection worked well, as the experiments were conducted in laboratory lighting, although some shielding may still be needed if strong light is used for visual stimulation, as strong microscope lighting over the electrodes produced significant noise. No benefit of the substrate passivation was immediately apparent from the data collected, suggesting that any benefit would be more likely to affect the system behind the shafts, and out of the immediate area of observation. Cross sections of the probe shafts revealed good coverage in SEMs, although failures would be most likely near the tip, where clean cross sections were difficult to make.

\section{CONCLUSION}

We have presented a new method for fabricating active neural probes using a standard CMOS process followed by a decoupled micromachining process and demonstrated their utility for in vivo measurements. The electronics on the probe have provided lownoise buffering and demonstrated an effective method for electrode offset cancellation that will be useful for systems designed with on-chip amplifiers. Finally, the substrate light protection implants enable experiments to be conducted in brightly lit conditions. Further work needs to be done to determine whether the substrate encapsulation realizes any benefit for the overall system, and future probe shaping would benefit from improved SOI etching techniques $[16]$.

\section{ACKNOWLEDGEMENTS}

This work has been funded by the National Science Foundation under grant \#PC147129-1. Additional support was provided by the NASA National Biocomputation Center.

\section{REFERENCES}

1. G. Buzsáki and A. Kandel, "Somadendritic backpropagation of action potentials in cortical pyramidal cells of the awake rat," J. Neurophysiol., vol. 79, pp. 1587-1591, 1998.

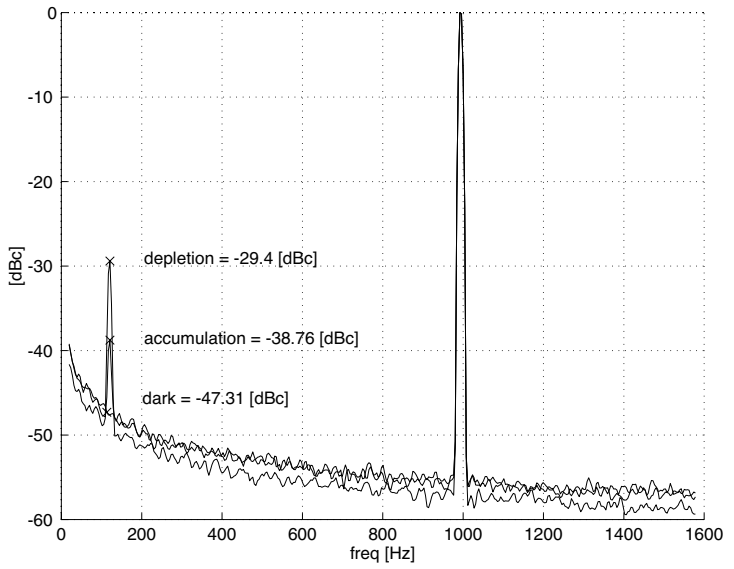

Figure 7. Spectral output of electrode in saline when under illumination and driven by a $\mathrm{Ag} / \mathrm{AgCl}$ electrode with a $\operatorname{lm} V_{P P}$ at $1 \mathrm{kHz}$ signal. The spectra provide a baseline measurment taken in the dark, and then demonstrate the improvement provided by the light protection implant (accumulation) compared to the (depletion) biasing conditions.

2. D. J. Edell, V. V. Toi, V. M. McNeil, and L. D. Clark, "Factors influencing the biocompatibility of insertable silicon microshafts in cerebral cortex," IEEE Trans. Biomed. Eng., vol. 39, pp. 635-643, 1992.

3. K. Takahashi and T. Matsuo, "Integration of multimicroelectrode and interface circuits by silicon planar and threedimensional fabrication technology," Sensors and Actuators, vol. 5, pp. 89-99, 1984.

4. K. Najafi and K. D. Wise, "An implantable multielectrode array with oon-chip signal processing," IEEE J. Solid-State Circuits, vol. SSC-21, pp. 1035-1044, 1986.

5. Q. Bai and K. Wise, "Single-Unit Neural Recording with Active Microelectrode Arrays," IEEE Trans. Biomed. Eng., vol. 48, pp. 911-920, 2001.

6. M. D. Gingerich and K. D. Wise, "An active microelectrode array for multipoint stimulation and recording in the central nervous system," in Digest Int. Conf. on Solid-State Sensors and Actuators, pp. 280-283, 1999.

7. D. Kewley, M. Hills, D. A. Borkholder, I. Opris, N. Maluf, C. W. Storment, J. Bower, and G. T. A. Kovacs, "Plasma-etched neural probes," Sensors and Actuators A, vol. 58, pp. 27-35, 1997.

8. A. A. Ayón, R. Braff, C. C. Lin, H. H. Sawin, and M. A. Schmidt, "Characterization of a Time Multiplexed Inductively Coupled Plasma Etcher," J. Electrochem. Society, vol. 146, pp. 339349, 1999.

9. K. Cheung, G. Lee, K. Djupsund, Y. Dan, L. P. Lee, “A new neural probe using SOI wafers with topological interlocking mechanisms," in Proc. 1st Intl IEEE-EMBS Conf. on Microtech. in Med. \& Biology, 2000, pp. 507-511. 

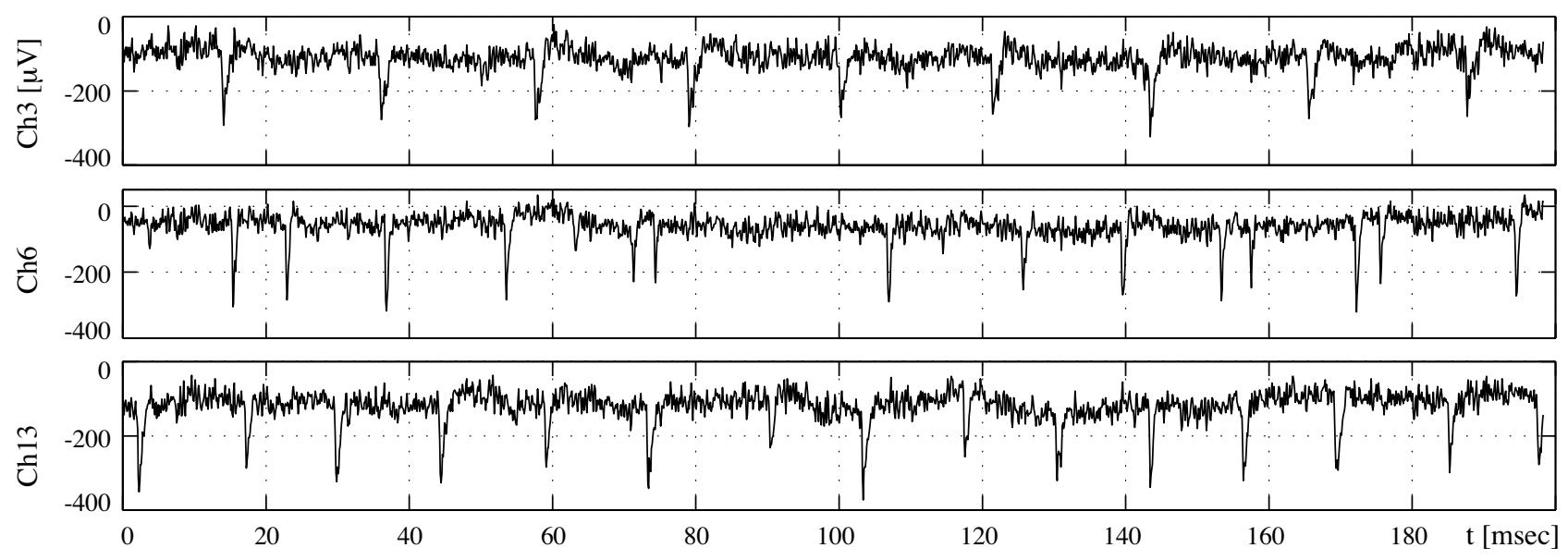

Figure 8. Recordings of spontaneous neural activity within the parietal cortex. The three traces shown are simultaneous recordings from an active probe in ambient lighting. The top trace was recorded from a site $160 \mu \mathrm{m}$ deeper in the cortex than the electrode corresponding to the central trace, while the bottom trace was from a site displaced $200 \mu \mathrm{m}$ laterally.

10. P. Norlin, M. Kindlundh, A. Mouroux, K. Yoshida, W. Jensen, and U. F. Hofmann, "A 32-site neural recording probe fabricated by double-sided deep reactive ion etching of silicon-on-insulator substrates," in Proc. Micromechanics Europe Workshop (MME2001), 2001, pp. 171-174.

11. A. Starr, K. D. Wise, and J. Csongradi, "An Evaluation of Photoengraved Microelectrodes for Extracellular Single-Unit Recording," IEEE Trans. Biomed. Eng., vol. BME-20, pp. 291-293, 1973.

12. K. D. Wise and J. B. Angell, "A low-capacitance multielectrode probe for use in extracellular neurophysiology," IEEE Trans. Biomed. Eng., vol. BME-22, pp. 212-219, 1975.

13. G. T. A. Kovacs, "Microelectrode models for neural interfaces," in Enabling Technologies for Cultured Neural Networks, D. A. Stenger and T. M. McKenna, Eds. San Diego:Academic Press, 1994, pp. 121-165.

14. A. Chandran, K. Najafi, and K. Wise, "A New DC Baseline Stabilization Scheme for Neural Recording Microprobes," in Proc. 1st Joint BMES/EMBS Conf., 1999, p. 386.
15. A. Partridge, J. Reynolds, J. Grade, B. Kane, N. Maluf, G. T. A. Kovacs, and T. Kenny, "An integrated controller for tunnel sensors," IEEE J. Solid-State Circuits, vol. 34, pp. 1099-1107, 1999.

16. C. Gormley, A. Boyle, V. Srigengan, and S. Blackstone, "HARM processing techniques for MEMS and MOEMS devices using bonded SOI substrates and DRIE," Proceedings of SPIE, vol. 4174, pp. 98-110, 2000.

17. W. Freiwald, A. Wannig, A. Kreiter, U. Hofmann, M. Hills, G. T. A. Kovacs, D. Kewley, J. Bower, C. Eurich, and S. Wilke, "Stimulus representation in rat primary visual cortex: multielectrode recordings and estimation theory," Neurocomputing, to be published.

18. K. H. Gilchrist, V. N. Barker, L. E. Fletcher, B. D. DeBusschere, P. Ghanouni, L. Giovangrandi, and G. T. A. Kovacs, "General purpose, field-portable cell-based biosensor platform," Biosensors and Bioelectronics, vol. 16, pp. 557-564, 2001. 